UNIVERSIDADE DE SÃO PAULO

FFCLRP - DEPARTAMENTO DE BIOLOGIA

PROGRAMA DE PÓS-GRADUAÇÃO EM ENTOMOLOGIA

A complexidade do substrato e o mesohabitat (remanso e corredeira) são fatores que influenciam a fauna de Chironomidae (Diptera) em riachos do Brasil Central?

Gustavo Rincon Mazão

Dissertação apresentada à Faculdade de Filosofia, Ciências e Letras de Ribeirão Preto da USP, como parte das exigências para a obtenção do título de Mestre em Ciências, Área: Entomologia

RIBEIRÃO PRETO -SP

2009 
UNIVERSIDADE DE SÃO PAULO

FFCLRP - DEPARTAMENTO DE BIOLOGIA

PROGRAMA DE PÓS-GRADUAÇÃO EM ENTOMOLOGIA

A complexidade do substrato e o mesohabitat (remanso e corredeira) são fatores que influenciam a fauna de Chironomidae (Diptera) em riachos do Brasil Central?

Gustavo Rincon Mazão

Orientador: Pitágoras da Conceição Bispo

Dissertação apresentada à Faculdade de Filosofia, Ciências e Letras de Ribeirão Preto da USP, como parte das exigências para a obtenção do título de Mestre em Ciências, Área: Entomologia

RIBEIRÃO PRETO -SP

2009 


\section{FICHA CATALOGRÁFICA}

\section{Gustavo Rincon Mazão}

A complexidade do substrato e o mesohabitat (remanso e corredeira) são fatores que influenciam a fauna de Chironomidae (Díptera) em riachos do Brasil Central?

Dissertação de Mestrado, apresentada à Faculdade de Filosofia, Ciências e Letras de Ribeirão Preto - USP, Área de concentração: Entomologia

Orientador: Pitágoras da Conceição Bispo

1.Complexidade. 2.Mesohabitat. 3.Insetos aquáticos. 4. Ambientes lóticos. 5.Cerrado. 


\section{Agradecimentos}

Redigir uma nota de agradecimento é sem dúvida uma das tarefas mais difíceis dentre tantas outras que realizei durante estes dois anos de estudos e trabalho. Difícil pelo fato de que não me perdoaria por ser injusto ao me esquecer de alguém que, de alguma maneira, do menor ao maior gesto, possa ter contribuído para a realização deste trabalho.

Agradeço à USP e à UNESP por terem me disponibilizado todo o apoio necessário, como infra-estrutura para o desenvolvimento do trabalho. Também agradeço à CAPES por ter me fornecido bolsa durante os dois anos de dedicação, pois sem esse dinheirinho, nada de mestrado concluído. À FAPESP (proc. 04/09711-8), que disponibilizou verba para a compra dos materiais e para os trabalhos de campo. Não posso me esquecer dos que me ajudaram a idealizar o projeto, e aqui fica meu respeito e admiração ao Dr. Luis Mauricio Bini e em especial ao "Seu" Pit, que mesmo sendo o Dr. Pitágoras da Conceição Bispo me permite tal proximidade. Não me canso de dizer Pit, além de exemplo de profissional gostaria de reforçar a pessoa maravilhosa que você é. Espero poder continuar aprendendo e crescendo com você. Saiba que sempre terá mais um braço, me prontifico a sempre ajudá-lo.

Devo agradecer especialmente a aqueles que me abrigaram sem ao menos me conhecerem previamente: Dona Angelina por sua aconchegante pensão, Gabriel Paciência por ter sido o primeiro a me alojar em Assis, Cristiano e Fausto que abriram as portas de suas casas para rápidas estadias em Ribeirão Preto e também aos meninos da Rep Label (André, Fabio, Kadu, Miuxa e Vitor) minha verdadeira casa nesses últimos tempos. 
Agradeço também a aqueles que colocaram a mão na massa: Davi, Srs. Aziane e Roberto por terem me ajudado com o tratamento das bolinhas de gude. Aos meus queridos amigos Tiago, Jujuba e Tati que me ajudaram com a montagem do experimento. Aqui vai um agradecimento especial à Jujuba, pois além de montar, voltou pra retirar o experimento, e não satisfeita, fez a análise físico-química da água nos pontos coletados. Aos três, saibam que os dias da minha coleta foram dias maravilhosos, pois estava em suas companhias.

Não poderia deixar de lado aqueles que não colocaram a mão na massa, mas pelo simples fato de serem pessoas especiais, me fortaleceram, dando força pra vencer meus obstáculos: primeiramente os meus familiares, aos meus queridos pais, Rubens e Zélia, minhas doces irmãs, Fabiane e Raquel, e a "cossa massi" linda do mundo minha Ana, eu os amo! Vocês são as pessoas mais valiosas do mundo. Aos meus avós paternos, Zezé e Bebé, e maternos, Nego e Magnólia, meus tios, tias, primos e primas um abraço muito especial. Cada um de vocês tem um espaço guardado no meu coração.

Neste segundo momento opto por não citar nomes, para não ser injusto citando uns antes de outros, até por que, sempre deixei claro a todos meus grandes amigos o quanto os respeito e admiro, sem distinções. Agradeço meus amigos de Goiânia, da minha época "metal" de bebedeiras e noites mal dormidas; meus amigos da bio UFG e LabLimno, que em meio a provas, trabalhos, coletas, relatórios de iniciação científica e horas de laboratório sempre encontrávamos momentos divertidos para boas conversas, cafezinhos da tarde, bete, filmes e alguns goles na Pamonharia; meus amigos de Ribeirão Preto dos laboratórios dos bentos, das abelhas, da genética, obrigado pelos cafés no seu Zé, cervejas na Cia da Esfirra e horas de aprendizado durante as disciplinas cursadas; amigos de Assis do meu querido Lábia, da bio, psico e letras UNESP, muito obrigado pelas pizzas no Santa Gula, almoços no "ruim", bete, lanches do Manigoto e 
Cebolinha, boliches, sinucas no Campeões do Taco, Dota, churrascos nos domingos e muito mais. Muito, mais muito obrigado mesmo a todos vocês! 


\section{ÍNDICE}

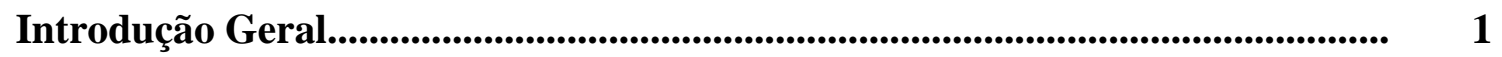

Referências Int. Ger....................................................................................... 2

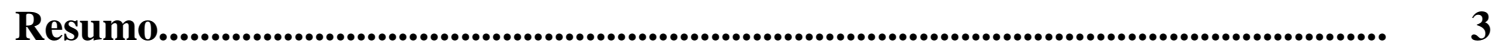

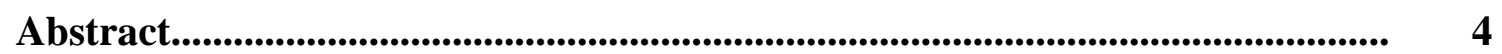

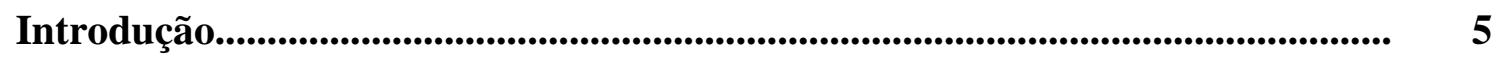

Materiais e Métodos..................................................................................... $\quad 7$

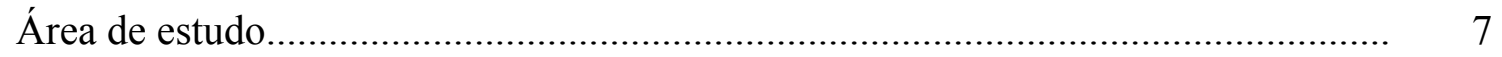

Caracterização ambiental.......................................................................... 8

Delineamento experimental........................................................................ 8

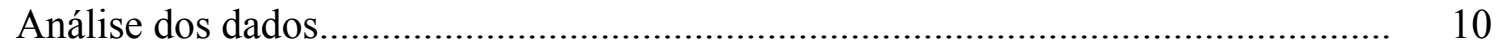

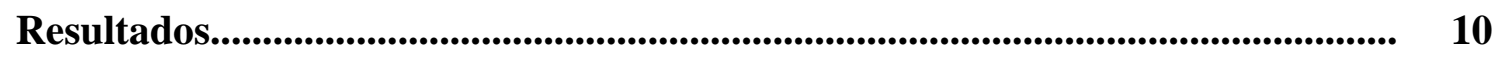

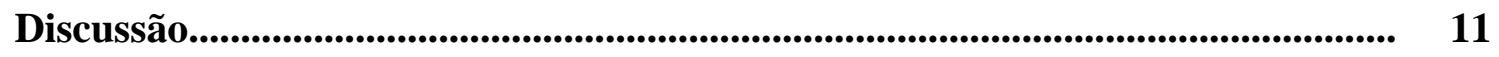

Agradecimentos............................................................................................................... 15

Referências Bibliográficas....................................................................................... 15

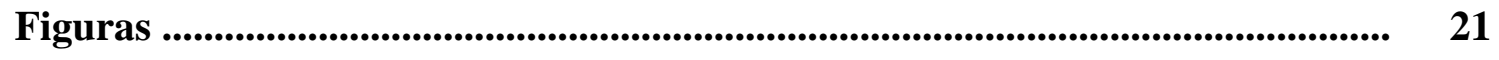

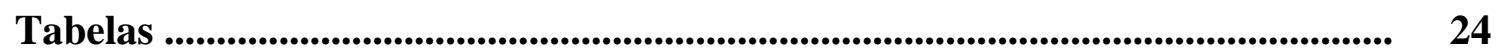




\section{Introdução geral}

O cerrado apresenta grande biodiversidade e vem sendo considerado um dos biomas mais prejudicados pela ocupação humana através do avanço agropecuário. Abrangendo boa parte do território brasileiro, este bioma é composto de uma série de fisionomias bastante variadas, Cerradão, Cerrado sensu stricto, Campos sujo e limpo além das veredas, campos rupestres e campos de murundu. O relevo, o tipo de solo, a ocorrência de fogo e a disponibilidade de água são alguns dos fatores determinantes das diferentes fisionomias vegetais observadas no cerrado. $\mathrm{O}$ alto endemismo, a grande complexidade e biodiversidade da biota ressaltam a importância do cerrado, definindo este último como área de destaque para conservação.

Os riachos que nascem no cerrado contribuem com a formação das principais bacias da América Latina. Estes ambientes possuem uma fauna característica com alta diversidade de espécies e muitas vezes sensível às perturbações ambientais. Portanto, é de grande importância que trabalhos sejam feitos em riachos com o objetivo de contribuir para o conhecimento da composição faunística, ecologia e biologia da biota, fornecendo informações relevantes para compreensão desse rico ecossistema e dando subsídios para um melhor manejo. Apesar do aumento do interesse e do avanço no conhecimento observados nos últimos 10 anos, ainda existem muitas lacunas no conhecimento sobre a diversidade e ecologia de organismos aquáticos em riachos de cerrado.

A fauna Chironomidae de riachos apresenta uma grande diversidade ecológica e de táxons, e tem recebido a atenção de vários pesquisadores nos últimos anos (Sanseverino \& Nessimian, 1998, 2001; Roque \& Trivinho-Strixino 2001; Roque et al., 2005). A grande diversidade e abundância de Chironomidae, a qual permite que teorias 
ecológicas sejam testadas através de seu estudo, a importância do cerrado e dos riachos como ambientes complexos e com alta diversidade motivaram a realização do presente trabalho. Somado a isso, o trabalho proporcionou a formação de coleção depositada no Laboratório de Biologia Aquática da Unesp de Assis, continuando procedimento já adotado, com finalidade de dar a referida coleção condições de ter uma amostra representativa de biodiversidade da entomofauna em ambientes lóticos.

Os resultados obtidos durante o desenvolvimento da presente dissertação são apresentados na forma de artigo.

\section{Referências Bibliográficas}

Roque, F. O.; Siqueira, T.; Trivinho-Strixino, S., 2005. Occurrence of chironimid larvae living inside fallen-fruits in Atlantic Forest streams, Brazil. Entomol. Vect. 12 (2): 275-282.

Roque, F. O. \& S. Trivinho-Strixino, 2001. Benthic macroinvertebrate in mesohabitats of different spatial dimensions in a first order stream (São Carlos - SP). Acta Limnologica Brasiliensia 13: 69-77.

Sanseverino, A. M. \& J. L. Nessimian, 2001. Habitats de larvas de Chironomidae

(Insecta, Díptera) em riachos de Mata Atlântica no Estado do Rio de Janeiro. Acta Limnologica Brasiliensia 13: 29-38.

Sanseverino, A. M. \& J. L. Nessimian, 1998. Habitat preferences of Chironomidae larvae in an upland stream of Atlantic Forest, Rio de Janeiro State, Brazil. Verhandlungen der Internationalen Vereinigung für Theoretische und Angewandte Limnologie 26: 2141-2144. 


\title{
A complexidade do substrato e o mesohabitat (remanso e corredeira) são fatores que influenciam a fauna de Chironomidae (Diptera) em riachos do Brasil Central?
}

\author{
Gustavo Rincon Mazão ${ }^{1} \&$ Pitágoras C. Bispo ${ }^{1}$
}

1. Laboratório de Biologia Aquática, Departamento de Ciências Biológicas, Faculdade de Ciências e Letras de Assis, Universidade Estadual Paulista. Avenida Dom Antônio 2100, 19806-900 Assis, São Paulo, Brasil.

E-mail: skullbio@usp.br

\section{Resumo}

O presente trabalho teve o objetivo testar os efeitos da complexidade do substrato e do tipo de mesohabitat (remanso e corredeira) sobre a riqueza, a abundância e a composição de Chironomidae (Diptera) em riachos. O experimento foi realizado em 12 riachos do Brasil Central, onde em cada mesohabitat (remanso e corredeira) foram colocadas duas bandejas de plástico, uma contendo substrato com alta complexidade e outra contendo substrato com baixa complexidade. Estes substratos permaneceram no riacho durante 20 dias. Os efeitos da complexidade do substrato e dos mesohabitats não foram significativos nem sobre a riqueza padronizada nem sobre a abundância, por outro lado, o efeito do mesohabitat foi significativo sobre a composição faunística. O primeiro eixo da DCA (33\% da variabilidade) separou claramente a fauna de remansos da de corredeiras. A análise de espécies indicadoras mostrou que Chironomus sp., Ablabesmyia sp.1, Ablabesmyia sp.2, Djalmabatista sp., Fittkauimyia sp., Labrundinia sp. indicaram o remanso, e Paratendipes sp., Polypedilum sp.2, Pentaneura sp., Rheotanytarsus sp.1, Corynoneura sp.2, Cricotopus sp.2, Lopescladius sp., Nanocladius sp.2, Parametriocnemus sp. e Thienemanniella sp. indicaram a corredeira.

Palavras Chaves: complexidade, insetos aquáticos, ambientes loticos, cerrado 


\begin{abstract}
Are the substrate complexity and the mesohabitat (pools and riffles) factors that influence the fauna of Chironomidae (Diptera) in streams of Central Brazil? The present study had the aim to test the effects of the substrate complexity and of the mesohabitat (pools and riffles) on the richness, abundance and composition of Chironomidae (Diptera) in streams. The experiment took place in 12 streams of Central Brazil. In each stream, two plastic trays were put in each one of the two studied mesohabitats (pool and riffle), one with higher complexity substrate and one with lower complexity substrate. These substrates were kept in the streams for 20 days. The effects of substrate complexity and of mesohabitat were not significative neither on the standarized richness nor on the abundance. On the other hand, the effect of mesohabitat was significative on the faunistic composition. The first axis of DCA $(33 \%$ of variability) separated cleanly the fauna in pools from those in riffles. The indicator species analysis showed that Chironomus sp., Ablabesmyia sp.1, Ablabesmyia sp.2, Djalmabatista sp., Fittkauimyia sp., Labrundinia sp. indicated pools, and Paratendipes sp., Polypedilum sp.2, Pentaneura sp., Rheotanytarsus sp.1, Corynoneura sp.2, Cricotopus sp.2, Lopescladius sp., Nanocladius sp.2, Parametriocnemus sp. and Thienemanniella sp. indicated riffles.
\end{abstract}

Key words: complexity, aquatic insects, lotics environments, cerrado 


\section{Introdução}

A estrutura e a dinâmica de populações e comunidades são determinadas em grande parte pelos efeitos dos fatores ambientais. Nesse sentido, as características químicas da água (e.g., a disponibilidade de nutrientes, a condutividade, o pH e o oxigênio dissolvido), o tamanho do riacho, a cobertura vegetal, as características hidráulicas, o tipo, a heterogeneidade e a complexidade do substrato são frequentemente levantados como fatores determinantes da riqueza e da distribuição espaço temporal dos organismos aquáticos em riachos (Vannote et al., 1980; Petersen \& Van Eeckhaute, 1992; Diniz-Filho et al., 1998; Bispo et al., 2006). Destes, certamente as características do substrato têm um efeito fundamental sobre os organismos bentônicos (Allan, 1975; Reice, 1980; Minshall, 1984; Pardo \& Armitage, 1997).

O tipo, a localização, a presença de refúgios e a complexidade do substrato afetam diretamente o risco de predação, a composição faunística, o tamanho dos indivíduos, a abundância e a riqueza das comunidades de macroinvertebrados aquáticos (Dudgeon, 1996; Lancaster, 1996; Taniguchi et al., 2003; Taniguchi \& Tokeshi, 2004; Gibbins et al., 2005; Costa \& Melo, 2008; Thomaz et al., 2008). Neste contexto, a manipulação experimental das características do substrato tem sido uma estratégia frequente para entender, o efeito de diferentes atributos do substrato sobre as comunidades de macroinvertebrados aquáticos (Taniguchi et al., 2003; Taniguchi \& Tokeshi, 2004). Entre as características manipuladas, está a complexidade do substrato, a qual pode ter um importante efeito sobre a diversidade de espécies.

Além das características do substrato, os fatores hidráulicos podem exercer um papel fundamental na estruturação das comunidades de macroinvertebrados aquáticos. Por exemplo, Doyle et al. (2005) demonstrou que variações de vazão, além de causar 
perturbações nas comunidades de macroinvertebrados, influenciam na disponibilidade de habitat, transporte da matéria orgânica, retenção de nutrientes e no crescimento populacional de algas. Aliado a isso, o tipo de substrato pode estar diretamente relacionado às características hidráulicas, já que locais com maior velocidade da água freqüentemente apresentam substrato rochoso. Por outro lado, locais com menor velocidade freqüentemente apresentam substrato formado por partículas menores como areia, argila, matéria orgânica particulada e folhas. O tipo de substrato e os fatores ligados à hidráulica como a velocidade, a vazão e o potencial erosivo são considerados alguns dos importantes fatores determinantes da riqueza, abundância e zonação de macroinvertebrados aquáticos em ambientes lóticos (Statzner \& Borchardt, 1994; Beisel et al., 1998; Crisci-Bispo et al., 2007). Corredeira e remanso são dois mesohabitats que diferem em relação à velocidade da água, profundidade e tipo de substrato dominante, o que em geral, podem levar a distintas composições faunísticas. Neste contexto, estudos experimentais considerando substrato com diferentes complexidades nos dois mesohabitats podem fornecer informações relevantes sobre o papel do substrato na estruturação da fauna de macroinvertebrados aquáticos.

Em riachos encontramos uma alta riqueza e abundância de macroinvertebrados aquáticos, dos quais a maior parte são insetos. Neste ponto, devido a sua representatividade nestes ambientes, damos devida atenção aos dípteros da família Chironomidae. Este grupo possui 355 gêneros distribuídos em 10 subfamílias (Ashe et al., 1987). As estimativas mundiais para o número de espécies de Chironomidae variam entre 10000 e 20000 (Coffman \& Ferrington, 1996). Além da alta riqueza e abundância, o grupo apresenta alta diversidade ecológica. Neste sentido, considerando a diversidade funcional, a maioria dos organismos pertencentes á família Chironomidae são herbívoro-detritívoros e raspadores, não nos esquecendo dos hábitos predadores 
representados em sua maioria pelos Tanypodinae (Nessimian \& Sanseverino, 1998). É importante salientar que apesar de ser possível caracterizar parte das espécies como pertencente a um determinado grupo funcional, baseado na estratégia alimentar dominante, muitos apresentam uma grande flexibilidade ingerindo uma ampla variedade de itens alimentares (Nessimian \& Sanseverino, 1998), portanto, muitos grupos podem ser considerados generalistas. Chironomidae é cosmopolita, ocorre na maior parte dos habitats aquáticos, e alguns ocorrem em habitats específicos como liteira do solo ou habitats marinhos (Trivinho-Strixino \& Strixino, 1995; Sanseverino \& Nessimiam, 2001).

No presente trabalho, dados experimentais sobre o efeito da complexidade do substrato e do mesohabitat (remanso e corredeira) sobre a fauna de Chironomidae foram obtidos em riachos do Brasil Central com o objetivo de responder a seguinte questão: a complexidade do substrato e o tipo de mesohabitat (remanso e corredeira) são determinantes da riqueza, da abundância e da composição de Chironomidae (Diptera) em riachos?

\section{Material e métodos}

\section{Área de estudo}

O presente estudo foi realizado em riachos dos municípios de Iporá, Ivolânida e Amorinópolis, no sudoeste do Estado de Goiás (Fig. 1). Esta região caracteriza-se por possuir como vegetação predominante pastagens, devido à intensa atividade de pecuária, além de cerrado sensu stricto nas áreas de conservação permanente. Na região há duas estações bem definidas: uma seca, que dura dos meses de maio a outubro, na qual há baixa incidência de chuvas, o que reduz drasticamente a vazão dos riachos, e 
outra chuvosa, dos meses de novembro a abril, na qual o volume de água dos riachos aumenta significativamente em decorrência das freqüentes chuvas. A montagem do experimento foi realizada no mês de agosto, e a retirada no mês de setembro de 2007, durante o período de seca.

\section{Caracterização ambiental}

Os seguintes fatores ambientais foram registrados nos riachos estudados: a ordem (Strahler, 1957), a temperatura da água $\left({ }^{\circ} \mathrm{C}\right)$, a velocidade $(\mathrm{m} / \mathrm{s})$ e a vazão $\left(\mathrm{m}^{3} / \mathrm{s}\right)$, o potencial hidrogeniônico $(\mathrm{pH})$, a condutividade elétrica $(\mu \mathrm{S} / \mathrm{cm})$, o oxigênio dissolvido (mg/l) e a turbidez (NTU). A velocidade da água foi medida usando um fluxômetro (Swoffer model 3000) e a vazão calculada através do produto da velocidade média da água por uma área de secção feita no riacho (Lind, 1979). A temperatura da água, a condutividade elétrica, o $\mathrm{pH}$ e o oxigênio dissolvido foram obtidos através do Analisador de Multiparâmetros Horiba, já a turbidez foi medida através de uma sonda (Hach model 2100P). Aliado a isso, as coordenadas e a altitude foram registradas através de um GPS. A caracterização dos locais onde o experimento foi realizado é apresentada na tabela 1 .

\section{Delineamento experimental}

$\mathrm{O}$ experimento foi realizado em 12 riachos de primeira a terceira ordem, segundo classificação de Strahler (1957). Em cada riacho foram mapeados habitats de remanso e de corredeira, sendo os ambientes de remanso caracterizados pela ausência de velocidade para o fluxômetro utilizado, e os de corredeira com velocidade acima de $0,255 \mathrm{~m} / \mathrm{s}$ (ver tabela 1). Em cada mesohabitat, duas bandejas de plástico foram colocadas, uma com substrato com alta complexidade (maior heterogeneidade) e uma 
com substrato com baixa complexidade (maior homogeneidade), paralelas entre si, sendo suas posições em relação à margem obtidas por meio de sorteio.

Cada bandeja teve $228 \mathrm{~cm}^{2}$ de área basal. O substrato com menor complexidade foi montado com bolinhas de gude de um único tamanho $(2 \mathrm{~cm}$ de diâmetro) e duas placas retangulares de concreto $(13 \times 3,5 \times 1 \mathrm{~cm})$ dispostas paralelas uma a outra, criando espaços similares entre as bolinhas. $\mathrm{O}$ substrato com maior complexidade foi montado com bolinhas de gude de dois tamanhos distintos ( 2 e $3 \mathrm{~cm}$ de diâmetro) e três cilindros de concreto ( $4 \mathrm{~cm}$ de altura e $4 \mathrm{~cm}$ de diâmetro) dispostos aleatoriamente, criando aleatoriamente espaços distintos entre as bolinhas (Fig. 2). O somatório da área superficial das bolinhas de gude assim como o das peças de concreto no dois tipos de bandeja (maior e menor complexidade) foram similares, excluindo assim, o viés da mudança da área superficial associada a mudança da complexidade do substrato. Antes da execução do experimento, as bolinhas de gude foram submetidas a um tratamento de choques físicos para torná-las mais ásperas.

Como os quironomídeos são organismos de rápida colonização, o substrato foi exposto durante 20 dias. Carvalho \& Uieda (2004) ressaltam que a abundância de quironomídeos permanece constante a partir de 20 dias de colonização. Após a colonização, os pares de bandejas foram removidos com o auxílio de uma rede de mão, tipo $\mathrm{D}$, com malha de $0,250 \mathrm{~mm}$. Tal procedimento foi adotado para que não houvesse perda de nenhum organismo. Após as bandejas serem removidas, as bolinhas e as peças de concreto foram lavadas em campo sendo todo material coletado devidamente armazenado em potes, etiquetados e fixados em formol $4 \%$.

Os organismos pertencentes à família Chironomidae foram identificados em nível de gênero e posteriormente identificados em nível de morfoespécies (seguramente reconhecíveis) utilizando para esta finalidade as chaves taxonômicas de Wiederholm 
(1983), Trivinho-Strixino \& Strixino (1995) e Coffman \& Ferrington (1996). Para a identificação, lâminas semi-permanentes utilizando Hoyer foram montadas. Os organismos coletados foram depositados na coleção de referência na UNESP (Assis).

\section{Análises de dados}

Os dados obtidos foram logaritmizados (base 10) antes das análises. A riqueza é influenciada pelo número de indivíduos coletados (Gotelli \& Graves, 1996), deste modo, foram utilizados os resíduos da regressão entre o $\log$ da abundância e o $\log$ da riqueza como medida de riqueza padronizada, sem efeito da abundância (Jacobsen, 2005). Os efeitos da complexidade do substrato (alta complexidade $x$ baixa complexidade) e do mesohabitat (remanso x corredeira) sobre a riqueza padronizada, a abundância e a composição faunística de Chironomidae foram testados utilizando uma ANCOVA. Como o tamanho do riacho influência as comunidades de macroinvertebrados aquáticos (Vannotte et al., 1980; Paller et al., 2006), a vazão foi

utilizada como covariável. A composição faunística foi avaliada utilizando a DCA (Análise de Correspondência Destendenciada). Os escores do primeiro eixo foram utilizados como variável resposta representativa da composição faunística na ANCOVA. Também foi realizada uma análise de espécies indicadoras (Dufrene \& Legendre, 1997) com o intuito de se estabelecer as espécies indicadoras de remanso e de corredeira.

\section{Resultados}

Um total de 4149 indivíduos foi coletado. A lista de morfoespécies coletadas é apresentada na tabela 2. Os efeitos dos mesohabitats e da complexidade do substrato 
não foram significativos nem para a riqueza padronizada (Mesohabitat $F=0,001$ e $p=0,969$; Substrato $F=0,212$ e $p=0,647$ ) nem para abundância (Mesohabitat $F=0,193$ e $\mathrm{p}=0,662$; Substrato $\mathrm{F}=0,603$ e $\mathrm{p}=0,441$ ) (Fig. 3; Tab. 3). Os resultados referentes aos escores da DCA (primeiro eixo) indicaram diferença significativa apenas para mesohabitat $(\mathrm{F}=47,821$ e $\mathrm{p}<0,001)$, não sendo significativo nem para a complexidade do substrato $(\mathrm{F}=0,73$ e $\mathrm{p}=0,397)$ e nem para as interações (Fig. 3; Tab. 3).

Os dois primeiros eixos da DCA explicaram juntos $44 \%$ da variabilidade. $\mathrm{O}$ primeiro eixo (33\% da variabilidade) separou claramente a fauna de remansos da de corredeiras (Fig. 4). Não foi observada a separação entre a fauna coletada nos substratos com baixa e alta complexidade. (Fig. 4). O segundo eixo, o qual explicou $11 \%$ da variabilidade, não pôde ser interpretado. A análise de espécies indicadoras mostrou que as morfoespécies Chironomus sp., Ablabesmyia sp.1, Ablabesmyia sp.2, Djalmabatista sp., Fittkauimyia sp., Labrundinia sp. indicaram o remanso, e Paratendipes sp., Polypedilum sp.2, Pentaneura sp., Rheotanytarsus sp.1, Corynoneura sp.2, Cricotopus sp.2, Lopescladius sp., Nanocladius sp.2, Parametriocnemus sp. e Thienemanniella sp. indicaram corredeira (Tab. 2).

\section{Discussão}

A complexidade do substrato é um importante determinante da diversidade local tanto da fauna de macroinvertebrados quanto de outros organismos aquáticos (Minshall, 1984; Taniguchi et al., 2003; Viera et al., 2007) atuando nas mais diversas formas, tanto influenciando nos padrões de deposição de partículas orgânicas e colonização de algas, quanto disponibilizando um maior número de refúgios e de nichos (Taniguchi \& Tokeshi, 2004). Neste sentido, o aumento da riqueza em locais com maior complexidade e heterogeneidade ambiental tem sido frequentemente documentado 
(Beisel et al., 2000; Taniguchi \& Tokeshi, 2004). Ao contrário do esperado, os nossos dados mostraram que não houve maior riqueza de Chironomidae no substrato com maior complexidade. Um dos fatores que poderia explicar este resultado é o fato da complexidade afetar a riqueza de organismos de diferentes maneiras, e a interação complexidade e riqueza certamente depende da escala e do tamanho dos indivíduos (Tolonen et al., 2003; Taniguchi \& Tokeshi, 2004). Por exemplo, Vieira et al. (2007), estudando comunidades perifídicas, verificaram que o efeito significativo da complexidade de folhas artificiais sobre a riqueza foi dependente do tamanho corpóreo. Portanto, acreditamos que a riqueza de Chironomidae seria afetada em níveis de complexidade em escalas menores, e que a escala criada no presente estudo, a partir da disposição de bolas de tamanhos similares (criado espaços homogêneos) e diferentes (criando espaços diferentes), foi demasiadamente grosseira para ter um efeito significativo sobre a riqueza de Chironomidae. Esta afirmação se apóia no fato dos indivíduos da família Chironomidae apresentarem um pequeno tamanho corpóreo quando comparados com outros grupos de macroinvertebrados de riachos. Aliado a isso, é importante salientar que usamos uma abordagem categórica (Taniguchi et al., 2003; Brown, 2007; Vieira et al., 2007) e atualmente uma abordagem da complexidade como variável contínua tem sido discutida como a melhor forma de obter informações mais acuradas (Taniguchi \& Tokeshi, 2004; Thomaz et al., 2008), e certamente novos estudos sobre o efeito da complexidade sobre a riqueza devem seguir esta linha.

Os riachos podem apresentar uma grande complexidade de habitats, sobretudo, devido ao efeito diferencial da velocidade da água sobre o substrato. Considerando os diferentes mesohabitats de um riacho, corredeira em geral apresenta substrato rochoso enquanto que o remanso apresenta substrato arenoso, comumente com acúmulo de matéria orgânica particulada e de folhas. Em regiões de corredeira, a disposição das 
pedras cria uma aparente complexidade e heterogeneidade (e.g. locais com e sem folhas retidas ou microhabitats sujeitos a diferentes pressões de correnteza) quando comparadas a regiões de remanso, onde aparentemente pode ser observada uma maior homogeneidade ambiental. Neste sentido, frequentemente, a maior complexidade aparente em regiões de corredeira tem sido utilizada para explicar a maior riqueza nestes locais (Uieda \& Gajardo, 1996; Buss et al., 2004). Por outro lado, nem todos os trabalhos indicam uma maior riqueza nas regiões de corredeira (Lind et al., 2006), mostrando que este não é um padrão universal. É importante salientar que a maior riqueza em corredeira pode não ser apenas o resultado da maior complexidade, já que há uma mudança na natureza do substrato (predominantemente rochoso em corredeira $\mathrm{x}$ predominantemente com areia e partículas de matéria orgânica em remanso). Além disso, frequentemente a maior riqueza em corredeira é associada a maior abundância nestes habitats, e isto por si só, já poderia aumentar a riqueza observada. Neste sentido, Crisci-Bispo et al. (2007) verificaram que a riqueza de EPT depois de padronizada pela rarefação para um mesmo número de indivíduos foi similar entre corredeira e remanso para o mesmo substrato (folhiço). Os dados do presente trabalho concordam com os dados de Crisci-Bispo et al. (2007) obtidos para EPT, já que não foi observada maior riqueza padronizada de Chironomidae em substratos colocados em corredeira quando comparados aos colocados em remanso.

Quanto à composição faunística, os resultados do presente trabalho apontam diferença significativa entre os dois mesohabitats. Remansos e corredeiras apresentam características ambientais diferentes, e diferentes composições faunísticas tem sido frequentemente registradas nestes dois mesohabitats. Este resultado concorda com os dados da literatura, os quais tem mostrado para diferentes grupos de macroinvertebrados aquáticos que, em geral, composições faunísticas distintas ocorrem nos diferentes 
mesohabitats (Sanseverino \& Nessimian, 1998; Amorim et al., 2004; Fenoglio et al., 2005). Dados não publicados sobre a fauna de EPT obtidos em riachos de Mata Atlântica (Bispo comunicação pessoal) mostram uma interação entre o tamanho do riacho e a diferenciação da fauna entre remanso e corredeira, em outras palavras, a distinção entre a fauna de remanso e de corredeira aumentou com a dimensão do riacho. Por outro lado, no presente trabalho a distinção entre a fauna de Chironomidae em remanso e corredeira permaneceu constante nos riachos estudados, independentemente da vazão.

Considerando as morfoespécies registradas, 15 (28\% do total de morfoespécies) foram indicadoras ou de corredeira (10) ou de remanso (5). Por outro lado, muitas das morfoespécies mais abundantes ocorreram nos dois mesohabitats com o número de indivíduos muito semelhante. É importante salientar que os quironomídeos são, em sua maioria, generalistas do ponto de vista alimentar (Nessimiam \& Sanseverino, 1998), além disso, são comuns em redes de deriva (Callisto \& Goulart, 2005; Hay et al., 2008), o que indica que a redistribuição destes organismos em ambientes lóticos pode ser comum. Estes dois fatores poderiam ser explicativos do porque parte das espécies abundantes foi encontrada nos dois mesohabitats. Assim como encontrado por Amorim et al. (2004), Corynoneura e Rheotanytarsus foram coletados predominantemente em corredeiras. Com exceção a Pentaneura sp., todo o restante dos Tanypodinae encontrados apresentou especificidade pelos ambientes de remanso, resultado semelhante ao encontrado por Roque \& Trivinho-Strixino (2001) em um riacho de primeira ordem. Sanseverino et al. (1998) também encontrou que os Orthocladiinae apresentaram preferência por ambientes de corredeira, neste sentido, os nossos dados mostram que Cricotopus sp.2, Lopescladius sp., Nanocladius sp.2, Parametriocnemus sp. e Thienemanniella sp. apresentaram preferência por este mesohabitat. 
Em resumo, as características distintas de remanso e de corredeira, não influenciaram a riqueza, por outro lado influenciaram a composição faunística. A homogeneização e a consequente diminuição da complexidade dos riachos pela ação antrópica pode ter efeitos drásticos sobre a diversidade de macroinvertebrados aquáticos, já que normalmente pode ser observada que grupos distintos de espécies estão associados aos diferentes mesohabitats (Pardo \& Armitage, 1997; Costa \& Melo, 2008). Neste sentido, a redução da complexidade relacionada aos mesohabitats pode diminuir a alta beta diversidade observada em riachos.

\section{Agradecimentos}

Os autores agradecem ao CNPq e a FAPESP pelo constante apoio. A CAPES e ao PPG em Entomologia pela bolsa de GRM. A Juliana Beatriz Souza Leite, Tatiana Raquel Alves Vilaça e Tiago Fernandes Carrijo pelo auxilio nos trabalhos de campo.

\section{Referências bibliográficas}

Allan, J. D., 1975. The distributional ecology and diversity of benthic insects in Cement Creek, Colorado. Ecology. 56: 1040-1053.

Amorim, R. M., A. L. Henrique-Oliveira, \& J. L. Nessimian, 2004. Distribuição espacial e temporal das larvas de Chironomidae (Insecta: Diptera) na seção ritral do rio Cascatinha, Nova Friburgo, Rio de Janeiro, Brasil. Lundiana 5: 119-127.

Ashe, P., D. A. Murray \& F. Reiss, 1987. The zoogeographical distribuction of Chironomidae (Insecta: Diptera). Annales, Limnologie 23: 27-60. 
Beisel, J. N., P. Usseglio-Polatera \& J. C. Moreteau, 2000. The spatial heterogeneity of a river bottom: a key factor determining macroinvertebrate communities. Hydrobiologia 422/423: 163-171.

Beisel, J. N., P. Usseglio-Polatera, S. Thomas \& J. C. Moreteau, 1998. Stream community structure in relation to spatial variation: the influence of mesohabitat characteristics. Hydrobiologia 389: 73-88.

Bispo, P. C., L. G. Oliveira, L. M. Bini \& K. G. Sousa, 2006. Ephemeroptera, Plecoptera and Trichoptera assemblages from riffles in mountain streams of Central Brazil: environmental factors influencing the distribution and abundance. Revista Brasileira de Zoologia 66: 611-622.

Brown, B. L., 2007. Habitat heterogeneity and disturbance influence patterns of community temporal variability in a small temperate stream. Hydrobiologia 586: 93106.

Buss, D. F., D. F. Baptista, J. L. Nessimian \& M. Egler, 2004. Substrate specificity, environmental degradation and disturbance structuring macroinvertebrate assemblages in neotropical streams. Hydrobiologia 518: 179-188.

Carvalho, E. M. \& V. S. Uieda, 2004. Colonization by benthic macroinvertebrates in artificial and natural substrates in a mountain stream from Itatinga, São Paulo, Brazil. Revista Brasileira de Zoologia 21: 287-293.

Callisto, M. \& M. Goulart, 2005. Invertebrate drift along a longitudinal gradient in a Neotropical stream in Serra do Cipo' National Park, Brazil. Hydrobiologia 539: 4756.

Coffman, W. P. \& L. C. Ferrington, 1996. Chironomidae. In: Merritt, R. W. \& K. W. Cummins (eds), An introduction to the aquatic insects of North America. Kendall, Hunt Publishing Co. Dubuque, IOWA: 635-754. 
Costa, S. S. \& A. S. Melo, 2008. Beta diversity in stream macroinvertebrate assemblages: among-site and among-microhabitat components. Hydrobiologia 598: $131-138$.

Crisci-Bispo, V. L., P. C. Bispo \& C. G. Froehlich, 2007. Ephemeroptera, Plecoptera and Trichoptera assemblages in two Atlantic rainforest streams, southeastern Brazil. Revista Brasileira de Zoologia 24: 312-318.

Diniz-Filho, J. A. F., L. G. Oliveira \& M. M. Silva, 1998. Explaining the beta diversity of aquatic insects in "cerrado" streams from Central Brazil using multiple Mantel Test. Revista Brasileira de Biologia 58: 223-231.

Doyle, M. W., E. H. Stanley, D. L. Strayer, R. B. Jacobson, \& J. C. Schmidt, 2005. Effective discharge analysis of ecological processes in streams. Water Resources Research 41: Art. No. W11411.

Dudgeon, D., 1996. The influence of refugia on predation impacts in a Hong Kong stream. Archiv für Hydrobiologie 138: 145-159.

Dufrene, M. \& P. Legendre, 1997. Species assemblages and indicator species: the need for a flexible asymmetrical approach. Ecological Monographs 67: 345-366.

Fenoglio, S., T. BO, P. Agosta \& M. Cucco, 2005. Mass loss and macroinvertebrate colonisation of fish carcasses in riffles and pools of a NW Italian stream. Hydrobiologia 532: 111-122.

Gibbins C. N., E. Scott, C. Soulsby \& I. Mcewan, 2005. The relationship between sediment mobilisation and the entry of Baetis mayflies into the water column in a laboratory flume. Hydrobiologia 533: 115-122.

Gotelli, N. J. \& G. R. Graves, 1996. Null Models in Ecology. Smithsonian Institution Press, Washington, DC. 
Hay, C. H., T. G. Franti, D. B. Marx, E. J. Peters \& L. W. Hesse, 2008. Macroinvertebrate drift density in relation to abiotic factors in the Missouri River. Hydrobiologia 598: 175-189

Jacobsen, D., 2005. Temporally variable macroinvertebrate-stone relationships in streams. Hydrobiologia 544: 201-214.

Lancaster, J., 1996. Scaling the effects of predation and disturbance in a patchy environment. Oecologia 107: 321-331.

Lind P. R., B. J. Robson, \& D. Michtell, 2006. The influence of reduced flow during a drought on patterns of variation in macroinvertebrate assemblages across a spatial hierarchy in two lowland rivers. Freshwater Biology 51: 2282-2295.

Lind, O. T., 1979. Handbook of Common Methods in Limnology. London, Cambridge The C. V. Mosby Company. 199.

Minshall, G. W., 1984. Aquatic insect-substratum relationships. In: Resh V.H. \& D. Rosenberg (eds), The Ecology of Aquatic Insects. Praeger, New York. 358-400.

Nessimian, J. L. \& A. M. Sanseverino, 1998. Trophic funtional characterization of Chironomidae larvae (Diptera: Chironomidae) in a first order stream at the mountain region of Rio de Janeiro State, Brazil. Verhandlungen der Internationalen Vereinigung für Theoretische und Angewandte Limnologie 26: 2115-2119.

Paller, M. H., W. L. Specht \& S. A. Dyer, 2006. Effects of stream size on taxa richness and other commonly used benthic bioassessment metrics. Hydrobiologia 568: 309316.

Pardo, I. \& P. D. Armitage, 1997. Species assemblages as descriptors of mesohabitats. Hydrobiologia 344: 111-128. 
Petersen, R. H. \& L. Van Eeckaute, 1992. Distributions of Ephemeroptera, Plecoptera, and Trichoptera of three maritime catchments differing in $\mathrm{pH}$. Freshwater Biology 27: $65-78$.

Reice, S. R., 1980. The role of substratum in benthic macroinvertebrate microdistribution and litter decomposition in a woodland stream. Ecological Society of America. 61: 580-590.

Roque, F. O. \& S. Trivinho-Strixino, 2001. Benthic macroinvertebrate in mesohabitats of different spatial dimensions in a first order stream (São Carlos - SP). Acta Limnologica Brasiliensia 13: 69-77.

Sanseverino, A. M. \& J. L. Nessimian, 2001. Habitats de larvas de Chironomidae (Insecta, Diptera) em riachos de Mata Atlântica no Estado do Rio de Janeiro. Acta Limnologica Brasiliensia 13: 29-38.

Sanseverino, A. M. \& J. L. Nessimian, 1998. Habitat preferences of Chironomidae larvae in an upland stream of Atlantic Forest, Rio de Janeiro State, Brazil. Verhandlungen der Internationalen Vereinigung für Theoretische und Angewandte Limnologie 26: 2141-2144.

Sanseverino, A. M., J. L. Nessimiam \& A. H. A. Oliveira, 1998. A fauna de Chironomidae (Díptera) em diferentes biótopos aquáticos na Serra do Subaio (Teresópolis, RJ). In: Nessimiam, J. L. \& A. L. Carvalho (eds) Ecologia de Insetos Aquáticos. Oecologia Brasiliensis 5: 253-263.

Statzner, B. \& D. Borchardt, 1994. Longitudinal Patterns and Process Along Streams: Modelling Ecological Responses to Physical Gradients. In: Giller, P. S., A. G. Hildrew \& D. G. Raffaelli (eds). Aquatic Ecology: Scale, Pattern and Process. British Ecological Society and Blackwell Science, Oxford, 113-140. 
Strahler, H. 1957. Quantitative analysis of watershed geomorphology. Transactions of the American Geophysical Union 8: 913-920.

Taniguchi, H. \& M. Tokeshi, 2004. Effects of habitat complexity on benthic assemblages in a variable environment. Freshwater Biology 49: 1164-1178.

Taniguchi, H., S. Nakano \& M. Tokeshi, 2003. Influences of habitat complexity on the diversity and abundance of epiphytic invertebrates on plants. Freshwater Biology 48: $718-728$.

Tolonen, K. T., H. Hamalainen, I. J. Holopainen, K. Mikkonen \& J. Karjalainen, 2003. Body size and substrate association of littoral insects in relation to vegetation structure. Hydrobiologia 499: 179-190.

Thomaz, S. M., D. Dibblee, L. R. Evangelista, J. Higuti, L. M. Bini, 2008. Influence of aquatic macrophyte habitat complexity on invertebrate abundance and richness in tropical lagoons. Freshwater Biology 53: 358-367.

Trivinho-Strixino, S. \& G. Strixino, 1995. Larvas de Chironomidae (Diptera) do Estado de São Paulo: Guia de Identificação e Diagnose dos Gêneros. PPG, ERN, UFSCar. 299.

Uieda, V. S. \& I. C. S. M. Gajardo, 1996. Macroinvertebrados perifíticos encontrados em poções e corredeiras de um riacho. Naturalia 21: 31-47.

Vannote, R. L., G. W. Minshall, K. W. L. Cummins, J. R. Sedell, \& C. E. Cushing, 1980. The River Continuum Concept. Can. J. Fish. Aquat. Sci. 37: 130-137.

Vieira, L. C. G., L. M. Bini, L. F. M. Velho \& G. R. Mazao, 2007. Influence of spatial complexity on the density and diversity of periphytic rotifers, microcrustaceans and testate amoebae. Fundamental and Applied Limnology 170: 77-85.

Wiederholm, T., 1983. The larvae of Chironomidae (Diptera) of the Holarctic region. Ent. Scand. Suppl., 19: 1-457. 


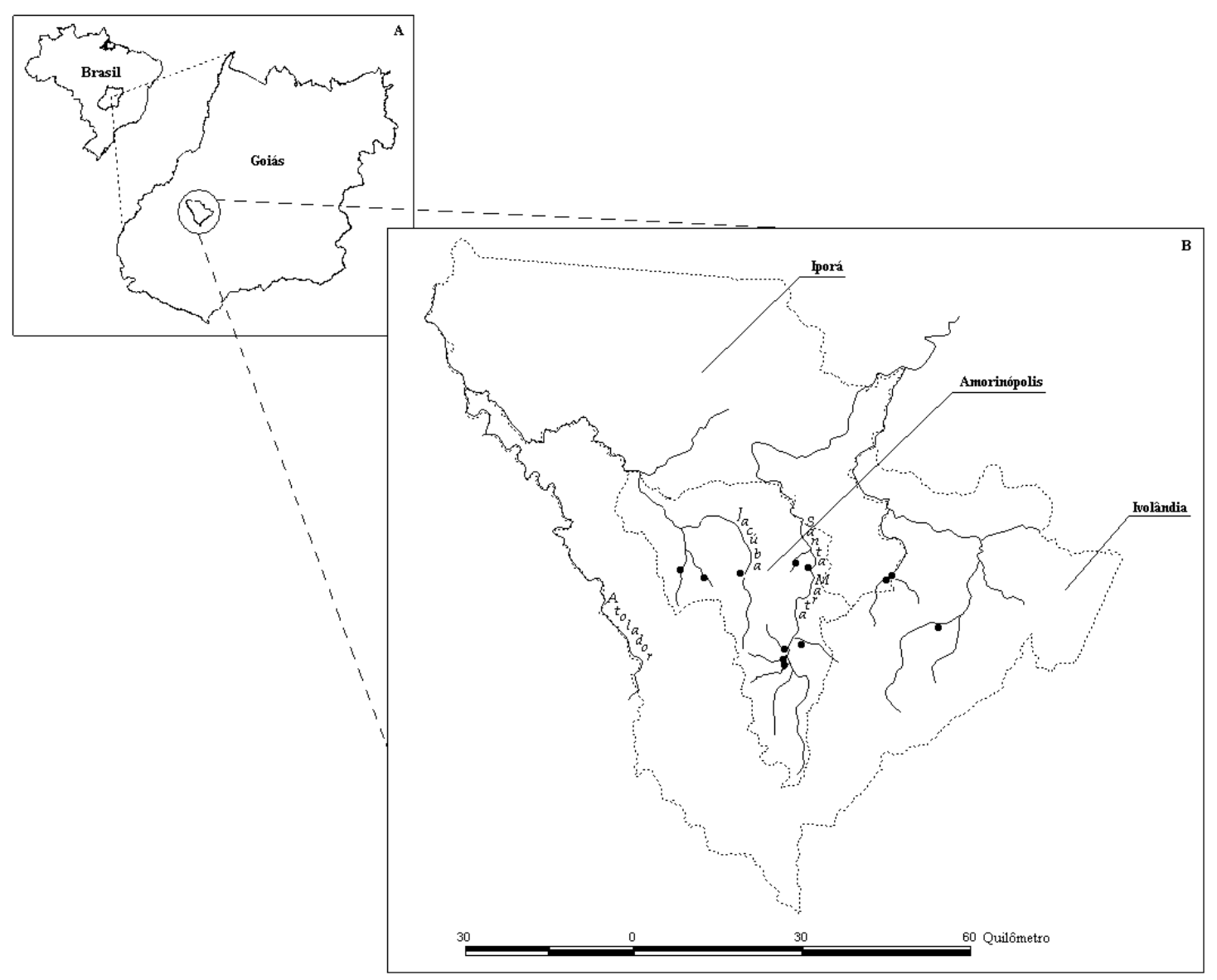

Fig. 1: A - Localização dos municípios estudados no sudoeste do estado de Goiás (Brasil). B - Riachos onde os experimentos foram realizados. Linhas pontilhadas, limites dos municípios de Iporá, Amorinópolis e Ivolândia.

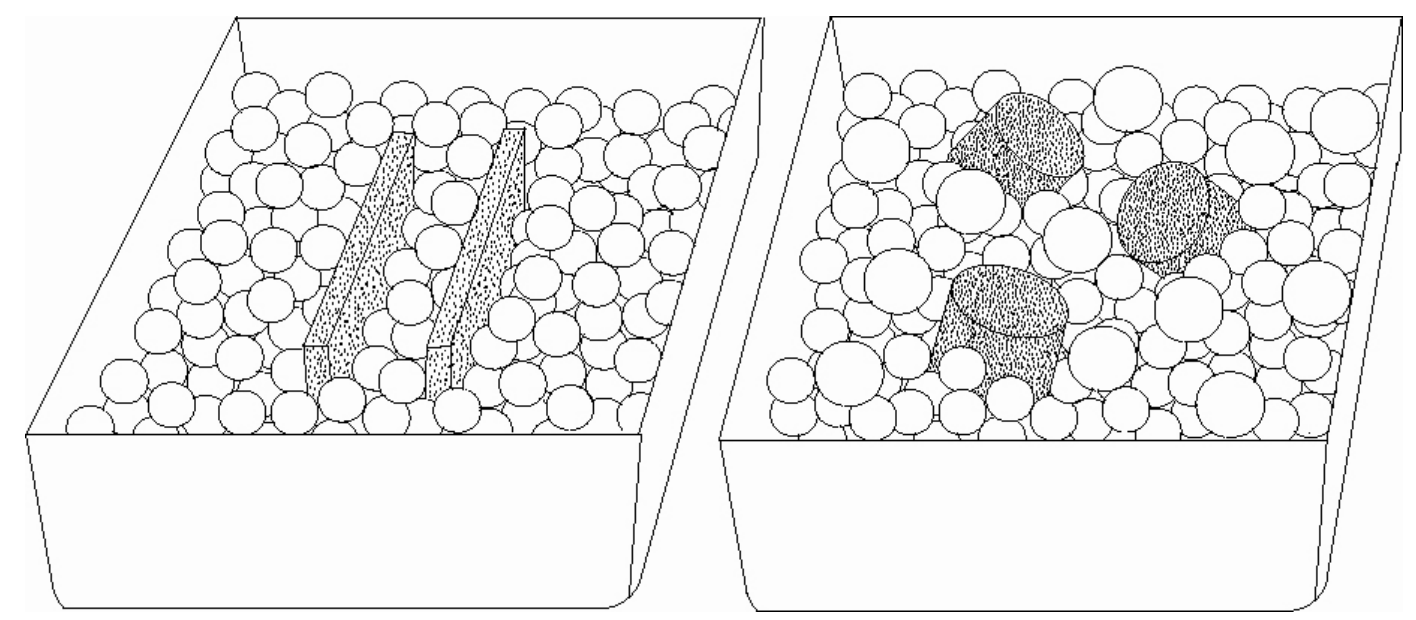

Fig. 2: Desenho ilustrativo representando as bandejas com substrato homogêneo (à esquerda) e heterogêneo (à direita). 

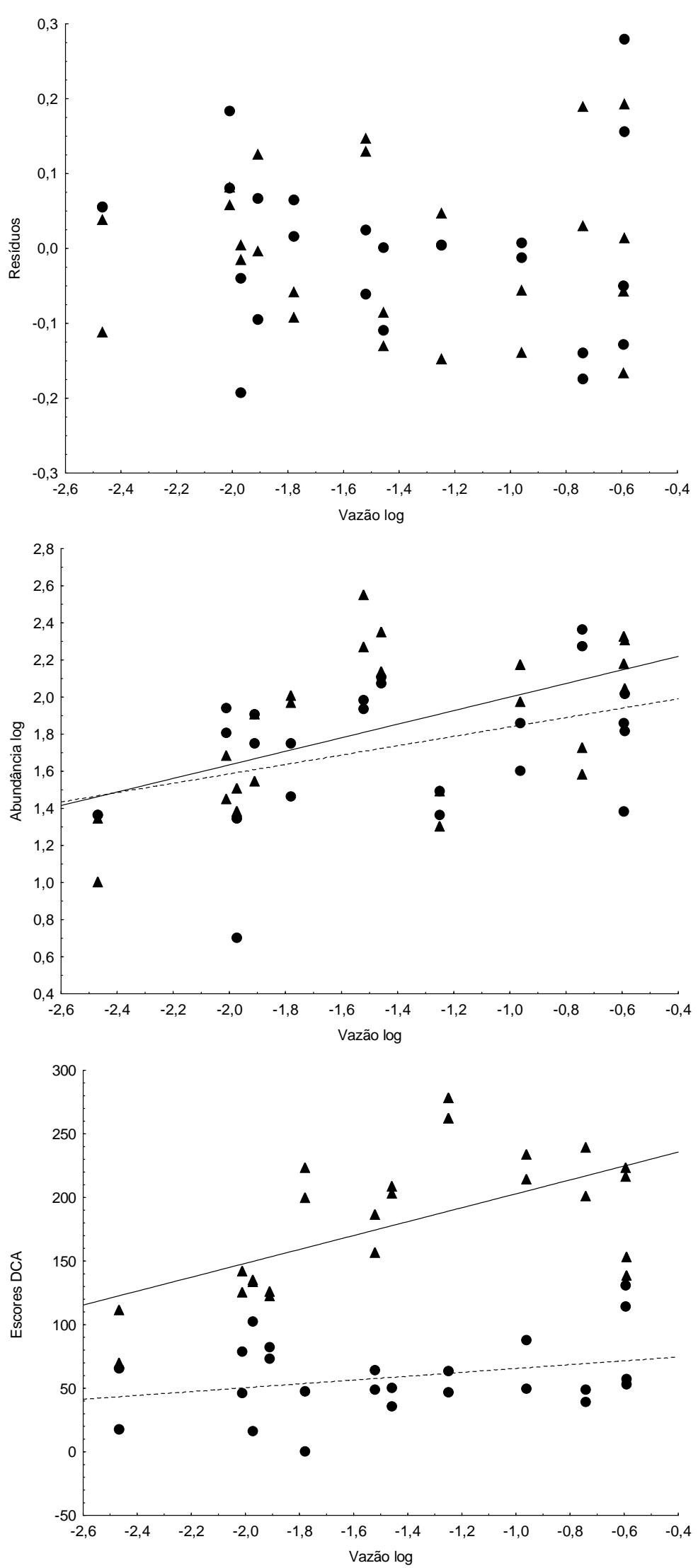

Fig. 3: Gráficos dos resultados da Ancova para os resíduos, abundância log e escores da DCA. Estão representados os pontos de remanso (---•---) e corredeira (- $\mathbf{A}-)$ em riachos dos municípios de Iporá, Amorinópolis e Ivolândia, Estado de Goiás, Brasil. 


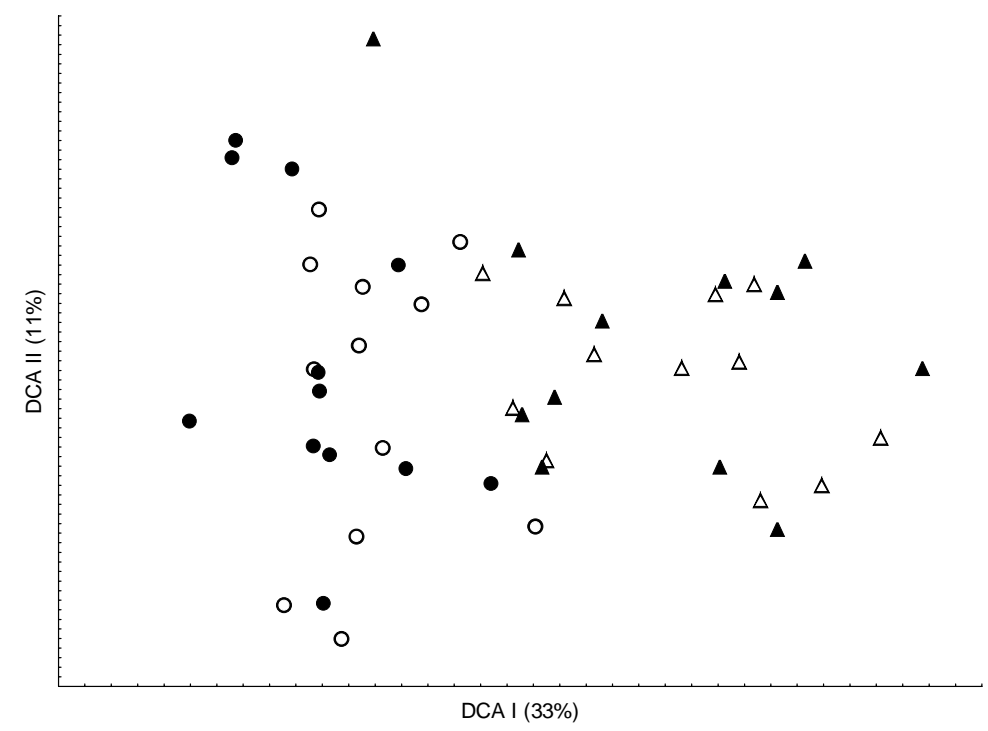

Fig. 4: Ordenação segundo a DCA da fauna de Chironomidae (Diptera) coletados nos substratos com baixa complexidade (símbolos vazios) e substratos com alta complexidade (símbolos preenchidos) em remanso (círculos) e em corredeira (triângulos) em riachos dos municípios de Iporá, Amorinópolis e Ivolândia, Estado de Goiás, Brasil. 
Tab. 1: Caracterização dos mesohabitats (remanso e corredeira) onde os experimentos foram realizados. 1-12 riachos dos municípios de Iporá, Amorinópolis e Ivolândia, Estado de Goiás, Brasil (R- remanso; C- corredeira).

\begin{tabular}{cccccccc}
\hline Pontos & DO mg/l & Turbidez & $\begin{array}{c}\text { Condutividade } \\
\text { MS/cm }\end{array}$ & $\begin{array}{c}\text { pH } \\
\text { 1R }\end{array}$ & $\begin{array}{c}\text { Temperatura } \\
{ }^{\circ} \mathrm{C}\end{array}$ & $\begin{array}{c}\text { Vazão } \\
\mathrm{m} / \mathrm{s} 2\end{array}$ & $\begin{array}{c}\text { Velocidade } \\
\mathrm{m} / \mathrm{s}\end{array}$ \\
\hline C C & 8,79 & 3 & 0,009 & 5,35 & 20,4 & 0,009768 & 0,000 \\
2R & 4,46 & 10 & 0,003 & 4,92 & 20,6 & 0,009768 & 0,255 \\
2C & 6,69 & 7 & 0,011 & 5,37 & 22,4 & 0,010714 & 0,000 \\
3R & 7,15 & 2 & 0,011 & 5,38 & 22,8 & 0,010714 & 0,496 \\
3C & 6,95 & 3 & 0,003 & 6,11 & 24,1 & 0,003411 & 0,000 \\
4R & 7,72 & 22 & 0,040 & 5,54 & 24,1 & 0,003411 & 0,445 \\
4C & 8,57 & 6 & 0,040 & 6,42 & 20,3 & 0,256998 & 0,000 \\
5R & 6,73 & 8 & 0,080 & 7,45 & 28,6 & 0,256998 & 0,345 \\
5C & 8,46 & 6 & 0,080 & 7,29 & 28,3 & 0,056383 & 0,000 \\
6R & 9,38 & 6 & 0,126 & 8,00 & 27,2 & 0,254963 & 0,300 \\
6C & 10,15 & 6 & 0,126 & 7,96 & 27,2 & 0,254960 & 0,000 \\
7R & 7,08 & 3 & 0,223 & 7,84 & 24,2 & 0,012357 & 0,000 \\
7C & 7,40 & 2 & 0,224 & 7,76 & 24,0 & 0,012357 & 0,415 \\
8R & 7,25 & 4 & 0,098 & 7,83 & 22,7 & 0,034911 & 0,000 \\
8C & 7,68 & 5 & 0,098 & 7,47 & 22,5 & 0,034911 & 0,431 \\
9R & 8,27 & 11 & 0,029 & 6,15 & 22,7 & 0,182218 & 0,000 \\
9C & 8,91 & 8 & 0,028 & 5,92 & 22,8 & 0,182218 & 0,301 \\
10R & 7,06 & 9 & 0,023 & 6,11 & 25,1 & 0,109620 & 0,000 \\
10C & 8,44 & 6 & 0,023 & 6,28 & 25,1 & 0,109620 & 0,741 \\
11R & 7,84 & 5 & 0,026 & 6,28 & 24,6 & 0,016632 & 0,000 \\
11C & 8,85 & 5 & 0,026 & 6,65 & 24,7 & 0,016632 & 0,534 \\
12R & 5,86 & 5 & 0,073 & 6,91 & 23,2 & 0,030186 & 0,000 \\
12C & 7,98 & 5 & 0,071 & 6,61 & 23,2 & 0,030186 & 0,860 \\
\hline
\end{tabular}


Tab. 2: Espécies indicadoras dos mesohabitats remanso e corredeira de riachos dos municípios de Iporá, Amorinópolis e Ivolândia, Estado de Goiás, Brasil. IV valor indicador (\%); p obtido através de permutação de Monte Carlo, 5000 permutações.

\begin{tabular}{|c|c|c|c|}
\hline Morfoespécies & Mesohabitat & IV $(\%)$ & $\mathrm{P}$ \\
\hline Beardius sp.1 & Remanso & 8,3 & 0,725 \\
\hline Chironomini sp. & Remanso & 23,1 & 0,198 \\
\hline Chironomus sp. & Remanso & 28,6 & 0,023 \\
\hline Cryptochironomus sp. & Remanso & 9,5 & 0,332 \\
\hline Dicrotendipes sp & Corredeira & 8,3 & 0,506 \\
\hline Endotribelos sp & Remanso & 4,2 & 1 \\
\hline Fissimentum sp & Remanso & 4,2 & 1 \\
\hline Pelomus (?) sp.1 & Remanso & 12,5 & 0,223 \\
\hline Pelomus (?) sp.2 & Remanso & 4,2 & 1 \\
\hline Pelomus (?) sp. 3 & Corredeira & 20,8 & 0,052 \\
\hline Manoa cf. sp. & Remanso & 8,3 & 0,491 \\
\hline Nilothauma sp. & Corredeira & 12,5 & 0,216 \\
\hline Parachironomus sp. & Remanso & 19,7 & 0,192 \\
\hline Paratendipes sp. & Corredeira & 33,2 & 0,002 \\
\hline Phaenopsectra sp. & Remanso & 35,9 & 0,544 \\
\hline Polypedilum sp.1 & Remanso & 36,7 & 0,275 \\
\hline Polypedilum sp.2 & Corredeira & 60,3 & 0,018 \\
\hline Polypedilum sp.3 & Corredeira & 15,6 & 0,368 \\
\hline Pseudochironomus (?) sp. & Remanso & 27,8 & 0,152 \\
\hline Robackia sp. & Corredeira & 4,2 & 1 \\
\hline Stenochironomus sp. & Corredeira & 32,1 & 0,146 \\
\hline Zavreliella sp. & Remanso & 4,2 & 1 \\
\hline Ablabesmyia sp.1 & Remanso & 52,4 & 0,006 \\
\hline Ablabesmyia sp. 2 & Remanso & 71,7 & 0,001 \\
\hline Coelotanypus sp. & Remanso & 8,3 & 0,477 \\
\hline Denopelopia sp. & Remanso & 4,2 & 1 \\
\hline Djalmabatista sp. & Remanso & 25 & 0,019 \\
\hline Fittkauimyia sp. & Remanso & 20,8 & 0,05 \\
\hline Labrundinia sp. & Remanso & 71,5 & 0,001 \\
\hline Larsia sp. & Corredeira & 13,4 & 0,883 \\
\hline Macropelopia sp. & Remanso & 4,2 & 1 \\
\hline Pentaneura sp. & Corredeira & 66 & 0,019 \\
\hline Pentaneurini sp. & Remanso & 4,2 & 1 \\
\hline Caladomyia sp.1 & Remanso & 24,8 & 0,206 \\
\hline Caladomyia sp. 2 & Remanso & 45,5 & 0,2 \\
\hline Caladomyia sp.3 & Remanso & 4,2 & 1 \\
\hline Caladomyia sp.4 & Remanso & 51,4 & 0,354 \\
\hline Rheotanytarsus sp.1 & Corredeira & 77,5 & 0,001 \\
\hline Rheotanytarsus sp.2 & Corredeira & 18 & 0,16 \\
\hline Stempellinella sp. & Corredeira & 5,4 & 0,864 \\
\hline Stempellina sp. & Remanso & 4,2 & 1 \\
\hline Tanytarsini sp. & Remanso & 42,6 & 0,316 \\
\hline Tanytarsus sp.1 & Remanso & 2,1 & 1 \\
\hline Tanytarsus sp.2 & Remanso & 4,2 & 1 \\
\hline Tanytarsus sp. 3 & Remanso & 4,2 & 1 \\
\hline Corynoneura sp.1 & Remanso & 13,9 & 0,555 \\
\hline Corynoneura sp.2 & Corredeira & 34,7 & 0,023 \\
\hline Cricotopus sp.1 & Corredeira & 4,2 & 1 \\
\hline Cricotopus sp.2 & Corredeira & 37 & 0,011 \\
\hline Lopescladius sp. & Corredeira & 25 & 0,028 \\
\hline Nanocladius sp.1 & Corredeira & 23,5 & 0,428 \\
\hline Nanocladius sp.2 & Corredeira & 25 & 0,021 \\
\hline Onconeura sp. & Corredeira & 28,6 & 0,064 \\
\hline Parametriocnemus sp. & Corredeira & 57,8 & 0,001 \\
\hline Thienemanniella sp. & Corredeira & 52,4 & 0,001 \\
\hline
\end{tabular}


Tab. 3: Análise de Covariância testando o efeito da complexidade do substrato (alta e baixa complexidade) e do mesohabitat (remanso e corredeira) utilizando a vazão como covariável sobre os resíduos (riqueza padronizada), abundância e composição faunística (primeiro eixo da DCA) de Chironomidae em riachos dos municípios de Iporá, Amorinópolis e Ivolândia, Estado de Goiás, Brasil.

\begin{tabular}{lrl}
\hline Fatores & \multicolumn{2}{l}{$\mathrm{p}$} \\
\hline Resíduos & & \\
Mesohabitat & 0,001444 & 0,969879 \\
Substrato & 0,212449 & 0,647351 \\
Vazão & 0,047898 & 0,827875 \\
Subs.*Vaz. & 0,126382 & 0,724081 \\
Meso.*Vaz. & 0,003300 & 0,954477 \\
Meso.*Subs. & 0,445156 & 0,508475 \\
Meso.*Subs.*Vaz. & 0,005260 & 0,942547 \\
& & \\
Abundância (log 10) & & \\
Mesohabitat & 0,1932 & 0,662609 \\
Substrato & 0,6031 & 0,441979 \\
Vazão & 5,9377 & $\mathbf{0 , 0 1 9 3 6 4}$ \\
Subs.*Vaz. & 0,1499 & 0,700716 \\
Meso.*Vaz. & 0,2228 & 0,639449 \\
Meso.*Subs. & 0,0551 & 0,815636 \\
Meso.*Subs.*Vaz. & 0,0969 & 0,757246 \\
& & \\
Escores DCA & & \\
Mesohabitat & 47,8211 & $\mathbf{0 , 0 0 0 0 0 0}$ \\
Substrato & 0,7306 & 0,397779 \\
Vazão & 4,9445 & $\mathbf{0 , 0 3 1 8 8 5}$ \\
Subs.*Vaz. & 0,0218 & 0,883453 \\
Meso.*Vaz. & 0,2107 & 0,648735 \\
Meso.*Subs. & 0,2439 & 0,624138 \\
Meso.*Subs.*Vaz. & 0,0379 & 0,846673 \\
\hline & &
\end{tabular}

